\title{
Laboratory measurements on core-scale sediment/hydrate samples to predict reservoir behavior
}

Timothy J. Kneafsey, Yongkoo Seol, George Moridis, Liviu Tomutsa, and Barry M. Freifeld Lawrence Berkeley National Laboratory

\begin{abstract}
Measurements on hydrate-bearing laboratory and field samples are necessary in order to provide realistic bounds on parameters used in numerically modeling the production of natural gas from hydrate-bearing reservoirs. The needed parameters include thermal conductivity, permeability, relative permeability-saturation(s) relationships, and capillary pressure-saturation(s) relationships. We have developed a technique to make hydrate-bearing samples ranging in scale from core plug-size to core-size in the laboratory to facilitate making these measurements. In addition to pressure and temperature measurements, we use $\mathrm{x}$-ray computed tomography scanning to provide high-resolution data providing insights on processes occurring in our samples. Several methods are available to make gas hydrates in the laboratory, and we expect that the method used to make the hydrate will impact the behavior of the hydrate sample, and the parameters measured.
\end{abstract}

\section{Introduction}

To accurately predict natural gas recovery from reservoirs containing gas hydrates, knowledge of both the reservoir properties and the properties and processes of hydrate dissociation is required. Data acquired from measurements on both natural and laboratory-made samples are needed to 
Kneafsey, Seol, Moridis, Tomutsa, and Freifeld

provide bounds on parameters used in modeling studies. We have developed a new technique to produce core-scale gas hydrate/sediment samples that facilitates the understanding reservoirscale hydrate processes. These core-scale samples bridge the gap between 1) the fundamental thermodynamic properties that are derived from small (sub-core sized) homogeneous samples, and 2) the properties that are needed for modeling at the reservoir scale, where the coupling of heat and mass transport must be considered. Investigations are performed to evaluate the potential of depressurization and thermal stimulation for recovering gas from naturally occurring hydrates; more specifically, we monitor thermal gradients and mass fluxes in core-sized samples that are undergoing externally applied changes in pressure and temperature.

An outstanding issue in hydrate laboratory research pertaining to production of gas from a reservoir is that of hydrate habit (where and how hydrate occupies the porous medium). Hydrate may be disseminated, with the hydrate finely distributed throughout the sample; nodular, with hydrate nodes up to $\sim 5 \mathrm{~cm}$ in diameter present within the sample; layered, with hydrate layers interspersed between porous medium layers and possibly containing dispersed hydrate; and massive (Sloan, 1998). At the pore scale, it is also important to know whether the hydrate surrounds mineral grains or is mostly present in the pore bodies is also important. Clearly, hydrate habit is expected to greatly affect the heat and mass transfer through the medium. Limited field data indicate that hydrate may be part of the medium structural skeleton, separating some mineral grains. More field data are needed to understand hydrate habit at sites of potential interest to natural gas production. 
Kneafsey, Seol, Moridis, Tomutsa, and Freifeld

Laboratory-made samples can be produced in various configurations in order to provide ranges of relevant parameter values for modeling applications. The most repeatable technique used for making hydrates in the laboratory is that of Stern et al., (1998) in which small grains of ice are melted in the presence of a guest molecule (e.g. methane) at high pressure. The resulting porous hydrate can be compressed into nearly nonporous masses suitable for making thermodynamic and physical property measurements. Hydrate made in this fashion has also been crushed and mixed with mineral grains to simulate hydrate in a porous medium. This technique will provide hydrate as part of the structural skeleton of the medium, and does not provide grain-to-grain contact throughout the medium. Using Stern's method, investigations with three possible hydrate configurations (nonporous, porous, and mixed with mineral grains) have provided significant information, however, additional techniques are needed to simulate the range of hydrate configurations found in nature.

Hydrate may also be formed from the interaction of a continuous gas phase with capillary-held water (Handa and Stupin, 1992). This method likely occurs in nature, and tends to result in hydrate cementing mineral grains together (Winters et al., 2004). Using this method, we have made disseminated methane hydrate in a porous medium while attempting to maintain the mineral grain-to-grain contact that would be expected in a porous medium under a significant lithostatic load. Hydrate in this cementing disseminated configuration may increase the thermal conductivity of the porous medium relative to the case in which hydrate is part of the structural skeleton, since, in the latter case, hydrate bridges the grains providing a pathway for the heat. In contrast, a sample with hydrate grains (or layers) interspersed between mineral grains (or layers) will have a lower overall thermal conductivity of the porous medium. 
Our goals include making measurements suitable for validation of numerical codes, and developing techniques for making measurements on natural samples. Measurements required include thermal conductivity, permeability, relative permeability, and the kinetics of hydrate formation and dissociation. To date, we have performed repeatability tests of hydrate formation in partially saturated sand, quantified the thermal or pressure-induced dissociation that resulted from depressurization and thermal stimulation, and have made permeability and relative permeability measurements of hydrate-bearing sediments.

Combined pressure and temperature measurements are critical for understanding hydrate behavior. When it is assumed that the sample is homogeneous and the gradients in temperature can be neglected, pressure and temperature measurements are usually adequate to interpret laboratory test results. In some tests, however, heterogeneity is present within the samples, and a method that provides spatial information throughout the sample is needed. We use x-ray computed tomography (CT) scanning to provide high resolution data on processes occurring in our samples caused by the changing presence of hydrate in the sample. CT is valuable for ascertaining the sample uniformity, and for observing changes within the samples. Figure 1 shows the variation in samples made using Stern's method and by Handa's method. Each of the two layers of the sample in Figure 1a was visually homogeneous, however when characterized by $\mathrm{CT}$, density variations are very apparent, and a fracture is apparent near the upper left corner. In Figure $1 b$, the initial porosity and water saturation in the sample was quite uniform, however on hydrate formation, water migration caused hydrate saturation variations in the sample. 
Kneafsey, Seol, Moridis, Tomutsa, and Freifeld

\section{Hydrate Formation}

We start with sand, partially saturate it with water, and compact it into a vessel or flexible sleeve in which we maintain control over pressure and temperature. We bring our sample into the hydrate stability region (typically 7 to $10 \mathrm{MPa}$ and 3 to $5^{\circ} \mathrm{C}$ ), and allow the hydrate to form from the water held in the sand by capillarity in pendular rings and films. The typical time for hydrate to form is about 24 hours. Our samples sizes depend on the vessel and the experiment, with diameters ranging from 3.3 to $10.2 \mathrm{~cm}$ and 9 to $27 \mathrm{~cm}$ in length. We can produce samples with or without confining stress in steel vessels (Figure 2a) or vessels suitable for $\mathrm{x}$-ray computed tomography (Figure 2b). An example of a methane hydrate /sand sample and a pure methane hydrate sample is shown in Figure 3.

Once the methane hydrate has formed, a variety of tests can be performed including flow tests, inhibitor introduction, or thermal or pressure variation. An example of a single step in a multiple step thermal dissociation of a hydrate/sand sample is presented in Figure 4. In this test, thermocouples were located at the sample edge (inner edge of the pressure vessel), half radius, and center (Figure 2a). The temperature in the sample was changed by increasing the temperature of the bath in which the pressure vessel was submerged by $0.5^{\circ} \mathrm{C}$. Figure 4 shows that 0.16 moles of methane was released (of the approximately 1.9 moles of methane initially contained in the hydrate sample) as a response to the temperature increase causing a pressure increase of about $0.5 \mathrm{MPa}$ in the vessel. 


\section{Thermal Conductivity Estimation}

Data similar to these can be used to estimate the thermal conductivity of the sample (including the sand, hydrate, residual water, and gas) using numerical inversion. Figure 5 shows the use of inverse modeling to estimate the thermal conductivity of a sand/hydrate/water/gas system with water and hydrate saturations of 0.22 and 0.09 , respectively, providing a thermal conductivity of 2.16 W/mK. In this work, the inverse code iTOUGH2 was used (Finsterle, 1997). The temperature at the outer thermocouple was used as the driving force, and the thermal conductivity was automatically adjusted until the temperature at the center thermocouple matched the measured data.

The graphs on the left side of Figure 5a show the calibrated thermal conductivity for a temperature increase for the sand/water/gas system (top) and for the sand/hydrate/water/gas system (bottom), compared to the measured data. These calibrated responses fit the data very well for the calibrated thermal conductivities. The graphs on the right side of Figure 5a show the calculated thermal response of the system to a temperature decrease compared to the measured data. Again, the fit is excellent, providing verification of the calibrated values. Figure $5 \mathrm{~b}$ shows the calculated temperature response of the system for a number of thermal conductivity values compared to measured data. All of the calculated thermal responses fit the data to an extent, however, the calibrated value minimizes the difference between the measured data and computed response. The thermal conductivity estimated similarly for the sand/water/gas system was 2.63 $\mathrm{W} / \mathrm{mK}$. This reduction in thermal conductivity is in contrast to the values presented by Moridis et al, (2005) of $3.145 \mathrm{~W} / \mathrm{mK}$ for the sand/hydrate/water/gas system (water saturation of 0.19 , hydrate saturation of 0.36 ) and $2.38 \mathrm{~W} / \mathrm{mK}$ for the corresponding sand/water/gas system (water 
Kneafsey, Seol, Moridis, Tomutsa, and Freifeld

saturation of 0.52 ). The presence of hydrate affects the thermal conductivity of the medium. However, why the hydrate increases the thermal conductivity in one case and lowers it in the other is unclear.

Depressurization-induced dissociation tests show strong cooling due primarily to the endothermic nature of dissociation, and also to the Joule-Thompson effect (Figure 6). In Figure 6, a stable disseminated hydrate-in-sand sample was rapidly depressurized (gray curve) and then shut in (i.e. the gas was not allowed to vent from the system). Temperatures dropped rapidly to below the freezing point and produced ice as hydrate dissociated. As the sample rewarmed due to conduction from the surrounding bath, hydrate dissociation continued until the equilibrium condition (pressure and temperature) was again reached. At that time, the system was slowly depressurized causing dissociation of the remaining hydrate. Note that the temperature in the vessel center again passed through the freezing point causing the formation of ice before warming to the bath temperature.

\section{x-ray CT scanning}

X-ray computed tomography (CT) scanning has been used by a number of researchers in the study of gas hydrates. Uchida et al. $(2000,2003)$ used CT to investigate the types of hydrate found in the Mallik 2L-38 research well, and from the Nankai Trough. Miakmi et al. (2000) used CT to investigate hydrate dissociation in response to heating and depressurization in a sample from the same Mallik core. Hohnberg et al. (2003) used CT to assess the performance of a pressure coring and storage method showing successful storage over several months. Abegg et 
Kneafsey, Seol, Moridis, Tomutsa, and Freifeld

al. (2003) used CT to quantify the free gas in hydrate-bearing samples from Hydrate Ridge, off the Oregon (USA) coast.

We have used CT scanning to observe a number of hydrate-related phenomena. Using temperature, mass balance, and CT data, we observed the moving dissociation front caused by a one-dimensional thermal stimulus (Freifeld et al. 2002, Tomutsa et al. 2002). The sand/hydrate sample consisting of two sand-hydrate mixtures was cooled to the boiling liquid nitrogen temperature, placed in an equally cold vessel on top of a thermocouple, and then the top was exposed to ambient temperature. As the sample warmed, the hydrate began dissociating vertically downward. The gas emanated during dissociation was collected and quantified, and compared to the attenuation changes observed using CT. Good agreement was found between the CT attenuation changes and the rate and volume of gas collected.

Recently, we observed processes occurring during disseminated hydrate formation and dissociation in a partially water-saturated core-sized sand pack (Kneafsey et al., 2005). We observed water migration toward the hydrate-containing regions during both hydrate formation and dissociation. We attribute this to strong capillary pressure changes that occur when hydrate is present in the pore space, and hypothesize that the formation of hydrate partially fills the pores in the medium, making them appear smaller to the remaining water. The water is then is drawn through the pores by capillarity.

CT has been used to observe hydrate dissociation in a pipeline configuration. In this test, pure methane hydrate formed using the method of Stern et al, (1996) was placed in a horizontal 
Kneafsey, Seol, Moridis, Tomutsa, and Freifeld

cylindrical vessel, and the temperature was controlled from the outside (Gupta et al., 2005). We observed the hydrate formation from fairly uniformly packed granular ice and found the resulting hydrate to be nonuniform with a lower density region forming in the center. Water apparently flowed away from the center because as ice melted, the water was exposed to an increased capillary suction in the surrounding hydrate. The CT data were used to evaluate the usefulness of a heat transfer model in understanding dissociation in a pipeline showing the model to be adequate (Gupta, et al. 2005).

\section{Conclusions}

More measurements using laboratory-made and natural hydrate samples in various configurations are needed to better constrain numerical models of natural gas production from hydrate-bearing reservoirs. We have begun making samples of sufficient size and performing tests useful for model validation. We have begun measuring (or estimating from our data) thermal conductivity, permeability, relative permeability, and capillary pressure of hydratebearing samples.

Robust techniques are needed to examine and make measurements on painstakingly collected and preserved natural hydrate-bearing samples. These techniques must be developed through careful experimentation using well-characterized laboratory samples. We have routinely used CT to characterize our samples because it provides density information with good spatial resolution and is extremely useful for observing changes in samples. 
Kneafsey, Seol, Moridis, Tomutsa, and Freifeld Other hydrate formation techniques need to be devised, tested, and compared to natural samples. The hydrate formation technique will affect the parameters measured, and these effects are not quantified. Such quantification will require detailed examination of field and laboratory samples using a variety of methods including $\mathrm{CT}$, scanning electron microscopy, acoustic, nuclear magnetic resonance imaging, and other methods. 
Kneafsey, Seol, Moridis, Tomutsa, and Freifeld

\section{References}

Abegg, F., Freitag, J., Bohrmann, G., Brueckmann, W., Eisenhauer, A., Amann, H., Hohnberg, H.-J., "Free gas bubbles in the hydrate stability zone: Evidence from CT investigation under in-situ conditions", Geophysical Research Abstracts, Vol. 5, 10342, 2003

Finsterle, S., "ITOUGH2 User's Guide", 1997. Lawrence Berkeley National Laboratory Report LBNL-40400.

Freifeld, B.M., T.J. Kneafsey, L. Tomutsa, L.A. Stern, and S.H. Kirby, "Use of X-Ray Computed Tomographic Data for Analyzing the Thermodynamics of a Dissociating Porous Sand/Hydrate Mixture," Proceedings of the Fourth International Conference on Gas Hydrates, Yokohama, May 19-23, 2002, pp. 750-755

Gupta, A., E.D. Sloan, T.J. Kneafsey, L. Tomutsa, G. Moridis, "Modeling methane hydrate dissociation x-ray CT data using a heat transfer model", Fifth International Conference on Gas Hydrates, Trondheim, Norway, June 2005

Handa, Y.P. and Stupin, D., "Thermodynamic properties and dissociation characteristics of methane and propane hydrates in 70A radius silica gel pores", J. Physical Chemistry, 96: $8599,1992$.

Hohnberg, H.-J., H. Amann, F. Abegg, H. Mudrack, G. Bohrmann, M. Maggiulli, “Pressurized coring of near-surface gas hydrate sediments on Hydrate Ridge: The multiple autoclave corer, and first results from pressure core x-ray CT scans", Geophysical Research Abstracts, Vol. 5, 09128, 2003

Kneafsey, T.J., L. Tomutsa, G.J. Moridis, C.E. Taylor, and A. Gupta, "Methane Hydrate Formation and Dissociation in a Partially Saturated Sand - Measurements and Observations", Fifth International Conference on Gas Hydrates, Trondheim, Norway, June 2005 
Kneafsey, Seol, Moridis, Tomutsa, and Freifeld

Mikami, J., Y. Masuda, T. Uchida, T. Satoh, H. Takeda, "Dissociation of Natural Gas Hydrates Observed by X-ray CT Scanner", Annals of the New York Academy of Sciences 912:1011$1020,2000$.

Sloan, E.D., Jr., Clathrate hydrates of natural gases, second edition, revised and expanded. Marcel Dekker, NY, 1998, 754 pp.

Stern, L.A., Kirby, S.H. and Durham, W.B., "Polycrystalline methane hydrate: Synthesis from superheated ice, and low-temperature mechanical properties", Energy \& Fuels, 12(2): 2012111998.

Tomutsa, L., B. Freifeld, T.J. Kneafsey, and L.A. Stern, "X-ray Computed Tomography Observation of Methane Hydrate Dissociation", SPE Gas Technology Symposium held in Calgary, Alberta, Canada, 30 April-2 May 2002, SPE 75533, LBNL 49580

Uchida, T., S. Dallimore, and J. Mikami, "Occurrences of Natural Gas Hydrates beneath the Permafrost Zone in Mackenzie Delta: Visual and X-ray CT Imagery", Annals of the New York Academy of Sciences 912:1021-1033, 2000

Uchida, T., and A Waseda, "Origin and migration in gas hydrate-bearing sediments relevant to their subsurface occurrences in the Nankai Trough", Geophysical Research Abstracts, Vol. 5, 08071,2003

Winters, W.J., Pecher, I.A., Waite, W.F. and Mason, D.H., "Physical properties and rock physics models of sediment containing natural and laboratory-formed methane gas hydrate", American Mineralogist, 89(8-9): 1221-1227, 2004. 


\section{Figure Captions}

Figure 1. Uniformity of a sand/hydrate sample made using a. Stern's method. The top half of the porous sample is $60 \%$ sand, $40 \%$ hydrate, the lower half is $40 \%$ sand, $60 \%$ hydrate, b. Handa's method. The white rectangle on the right side is the thermocouple. Scales are density in $\mathrm{g} / \mathrm{cm}^{3}$. Figure2. a. Pressure vessel, bath, and thermocouple configuration. The bath is housed in a freezer. This system is not amenable to CT scanning. b. x-ray transparent vessel amenable to CT evaluation (Kneafsey et al., 2005).

Figure 3. Methane hydrate made in partially saturated sand (1) and pure methane hydrate (r). Scale is in inches.

Figure 4. Thermally induced dissociation step. Temperature in the bath (solid black), at the vessel/sample interface (large dash), half radius (mixed long and short dashes), center (small dash), and pressure (gray) recorded in thermal dissociation of $10 \mathrm{~cm}$ diameter, $11 \mathrm{~cm}$ long sand/hydrate sample.

Figure 5. Calibration of thermal conductivity for the sand-water-gas system (top-left) and the sand-hydrate-water-gas system (bottom-left) and validation of the calibrated thermal conductivity (right). Symbols are measured data and lines are simulated. b. Comparison of simulated temperatures with varying thermal conductivity for the two systems.

Figure 6. Pressure induced methane hydrate dissociation. Temperature in the bath (solid heavy black), at vessel/sample interface (large dash), half radius (mixed long and short dashes), center (small dash), and pressure (gray) recorded in pressure-induced dissociation of $10 \mathrm{~cm}$ diameter, $11 \mathrm{~cm}$ long sand/hydrate sample. The gas produced is shown in the thick mixed dash line on the right half of the figure (read on left axis). 


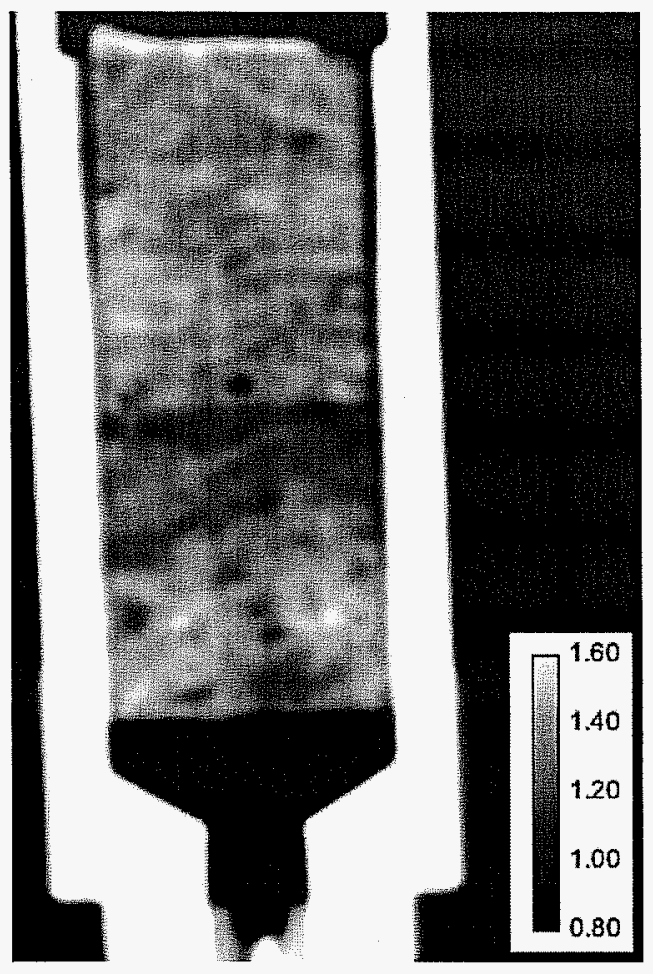

Figure 1a.

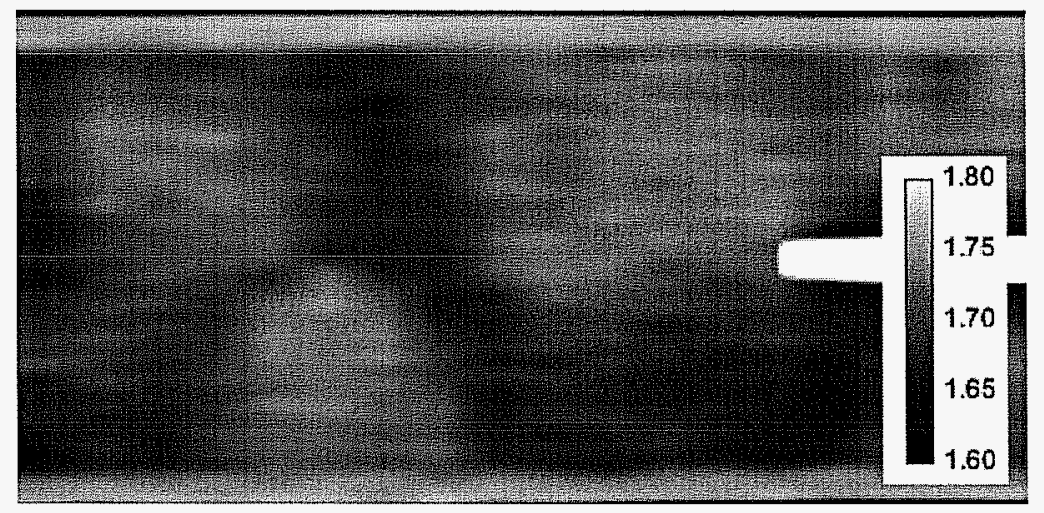

Figure $1 b$. 
Kneafsey, Seol, Moridis, Tomutsa, and Freifeld

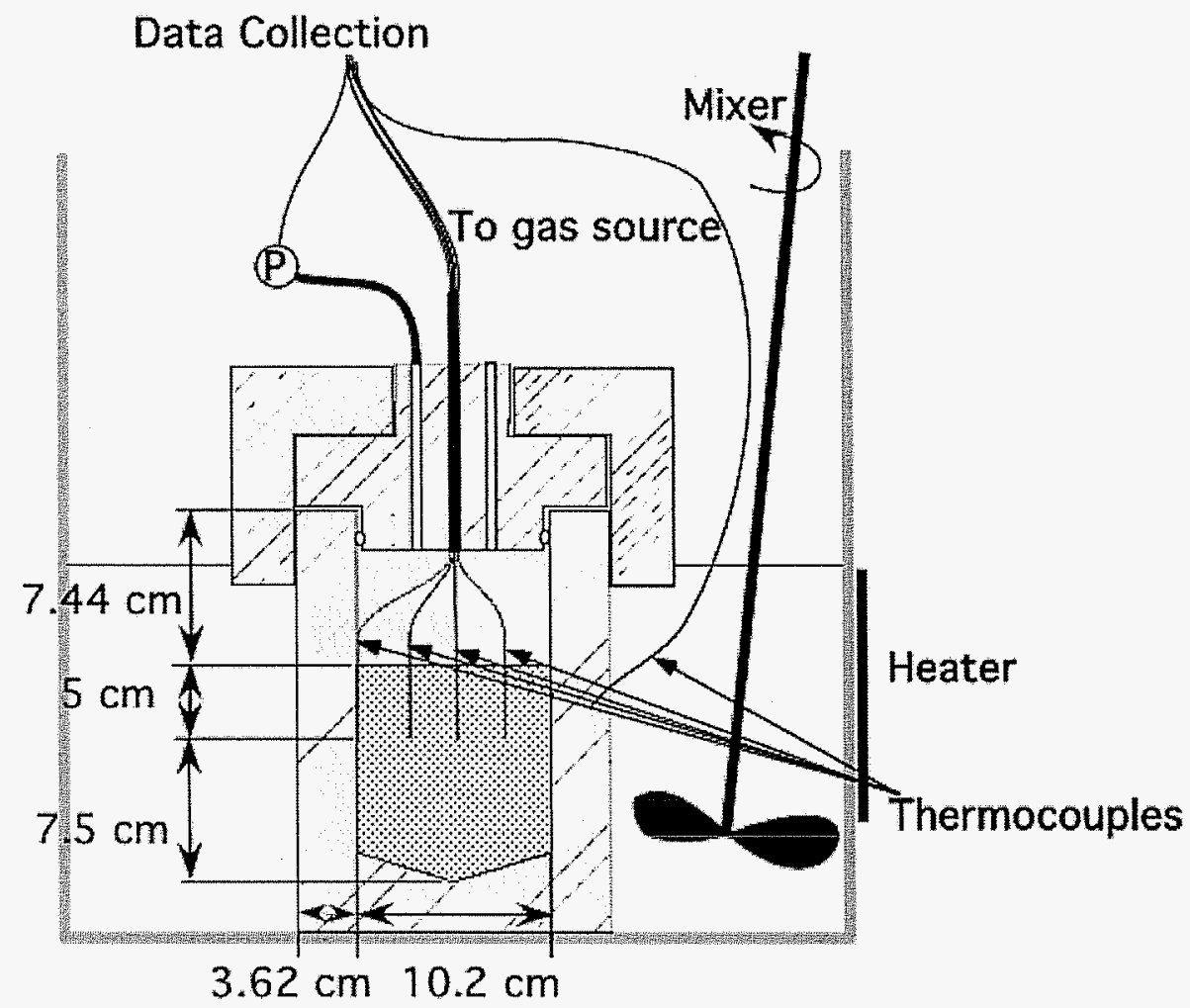

Figure 2a. 
Kneafsey, Seol, Moridis, Tomutsa, and Freifeld

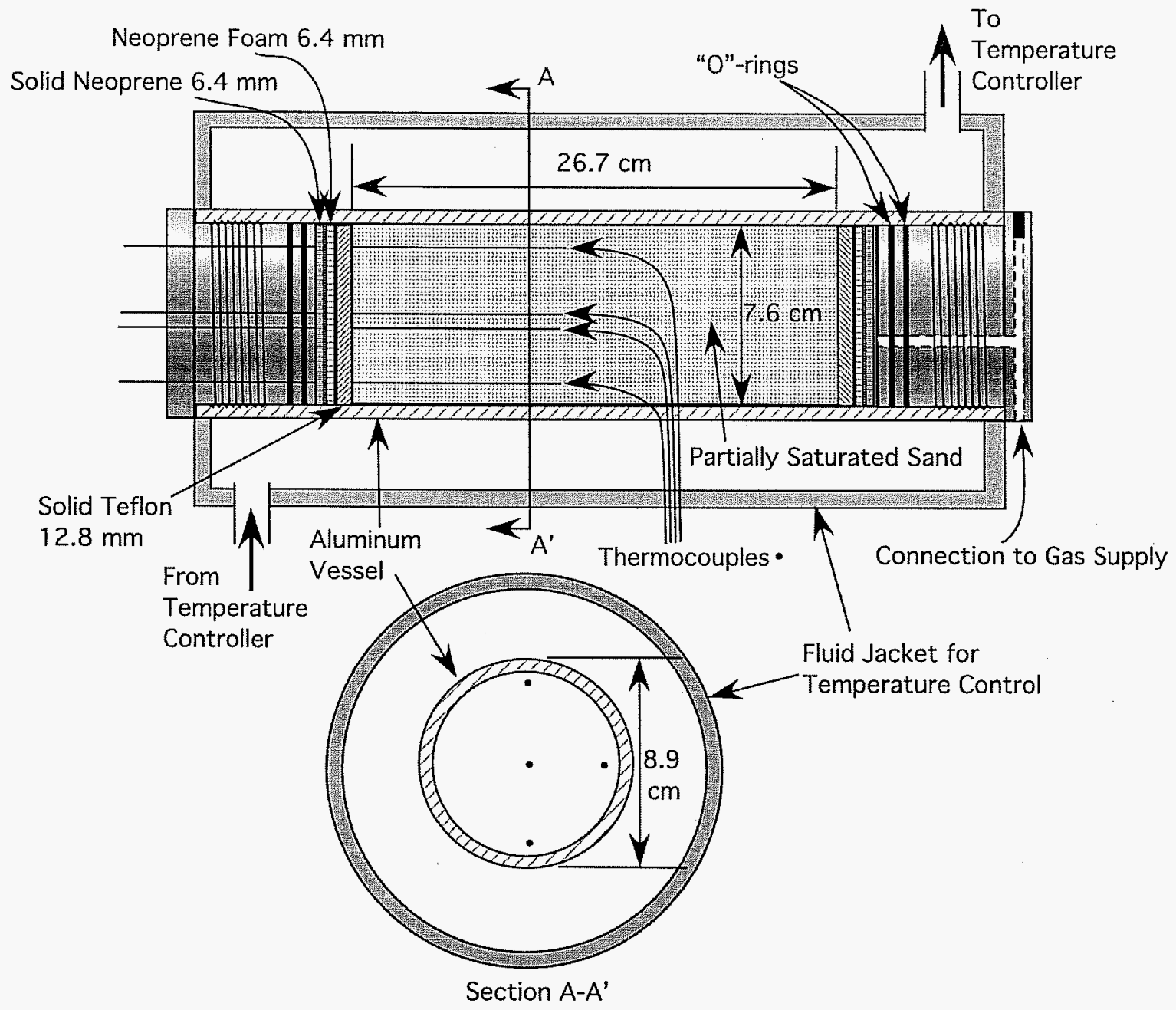

Figure $2 b$. 
Kneafsey, Seol, Moridis, Tomutsa, and Freifeld

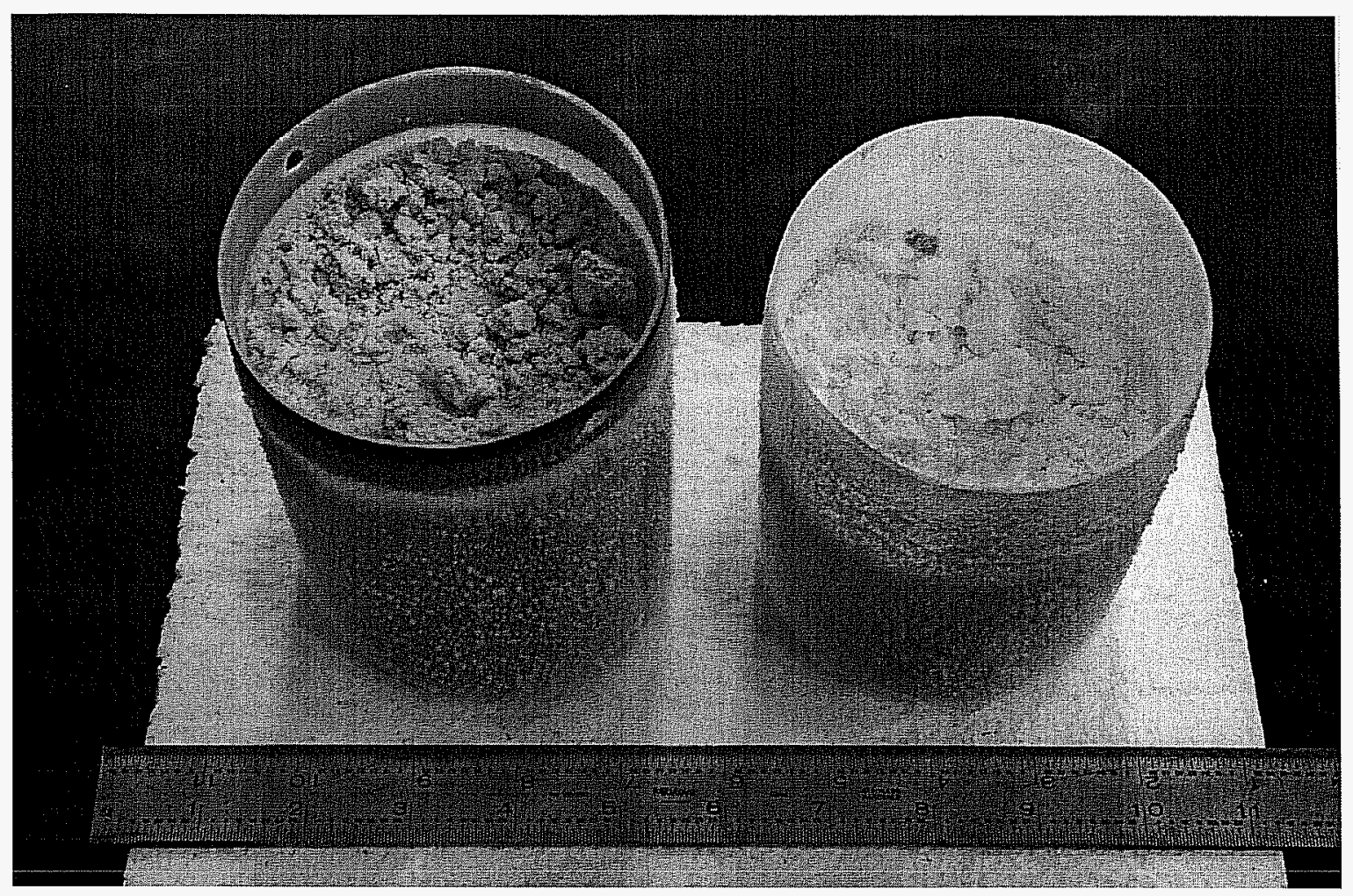

Figure 3. 


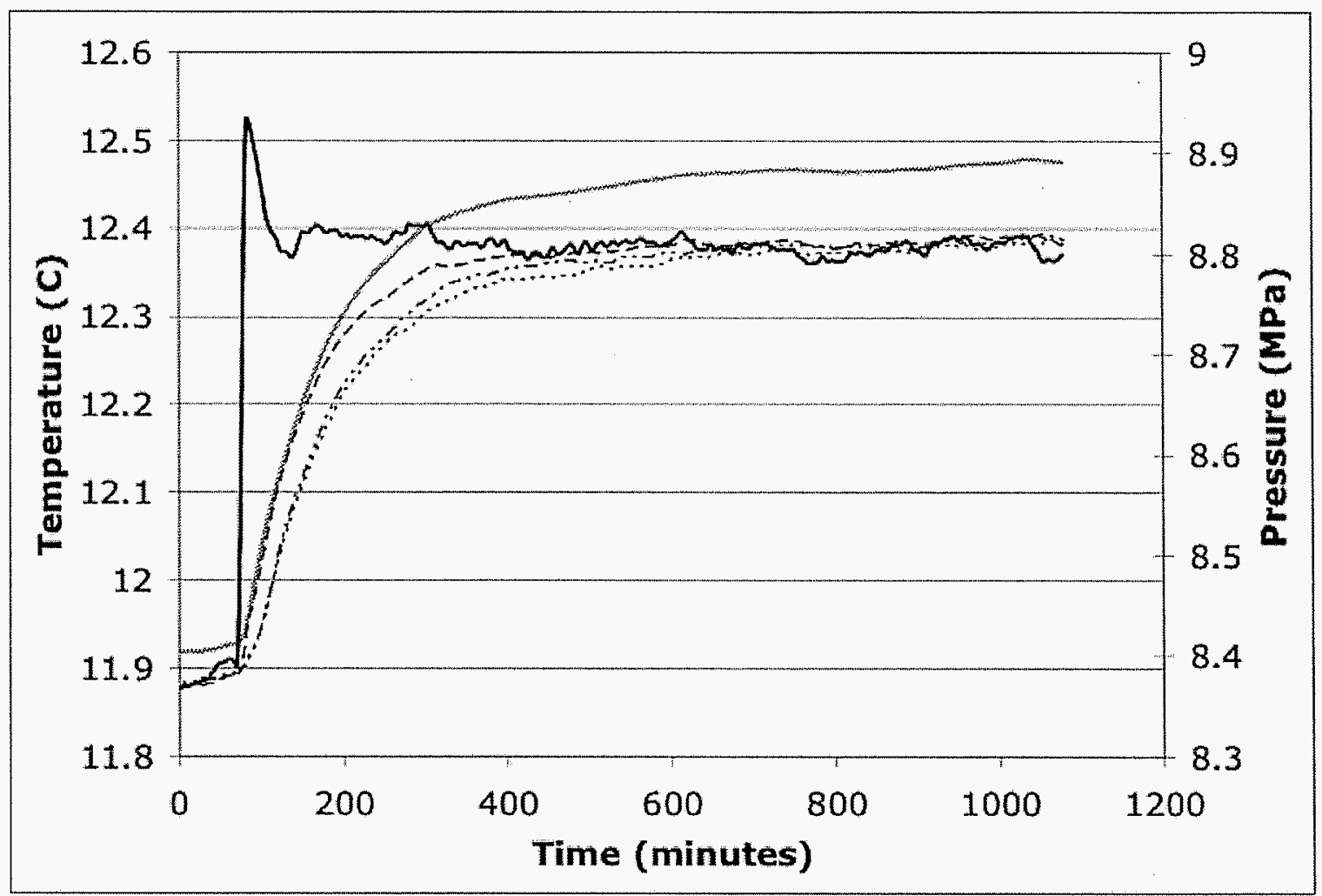

Figure 4. 
Kneafsey, Seol, Moridis, Tomutsa, and Freifeld
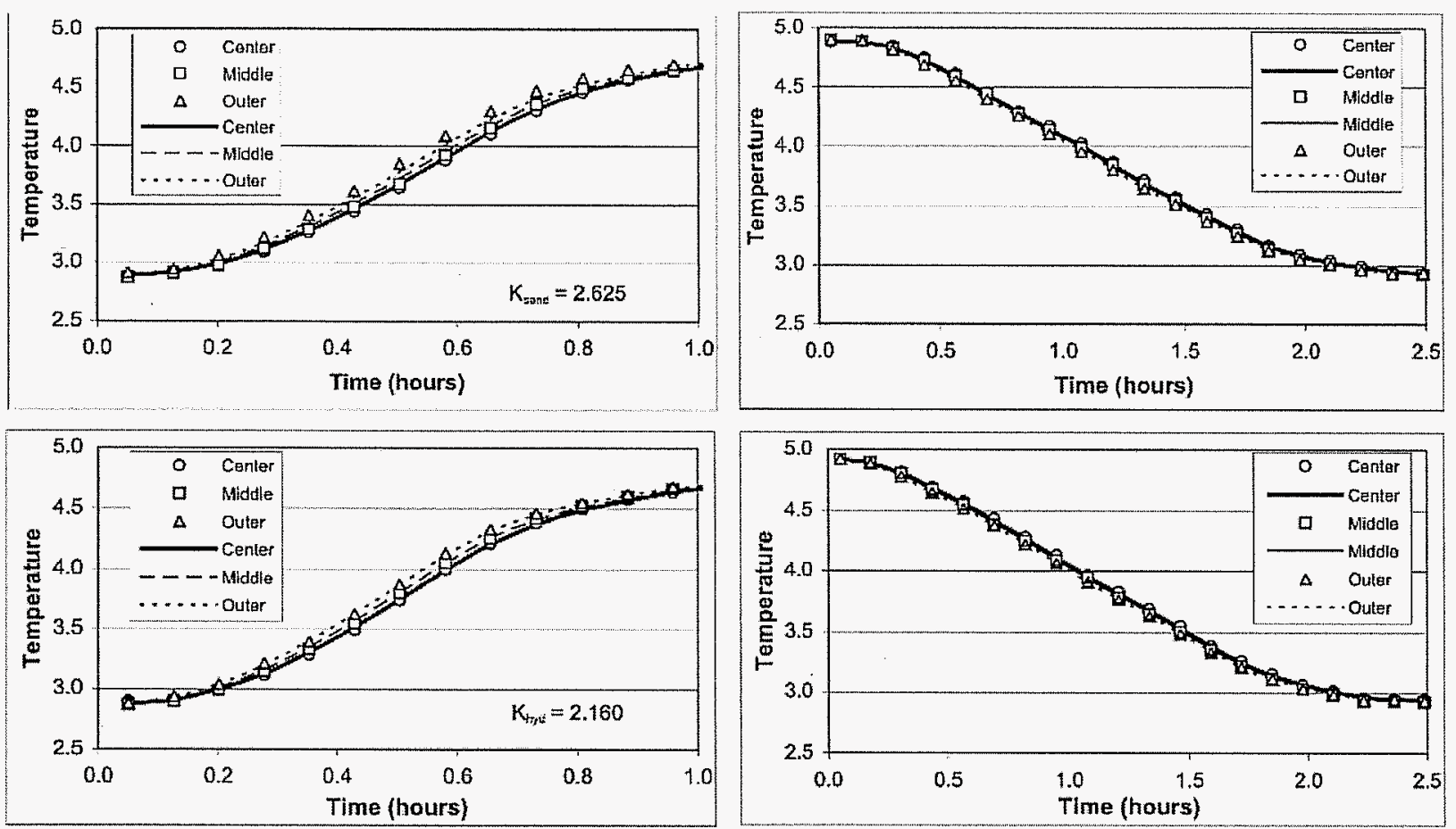

Figure 5a. 
Kneafsey, Seol, Moridis, Tomutsa, and Freifeld
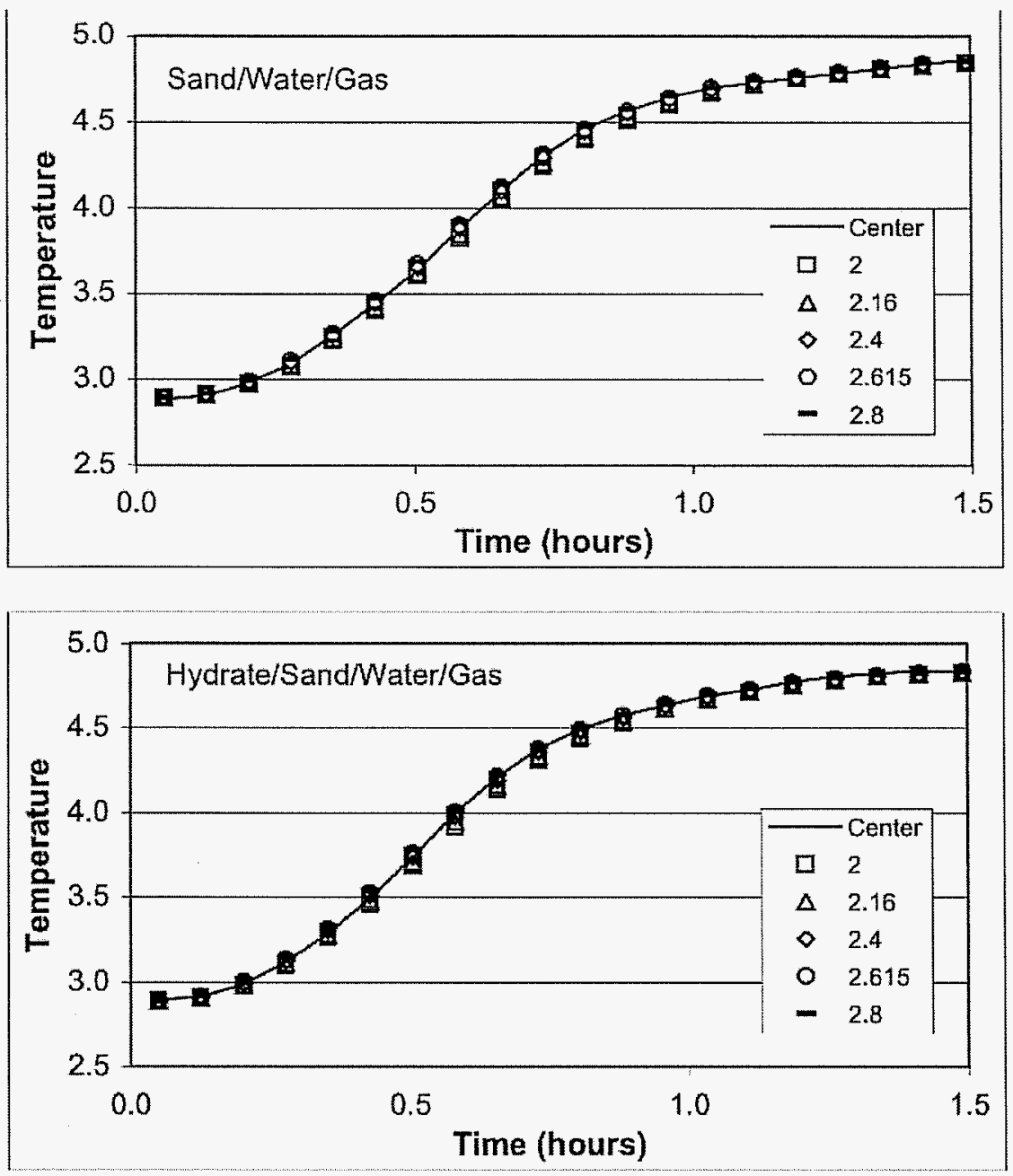

Figure 5b 
Kneafsey, Seol, Moridis, Tomutsa, and Freifeld

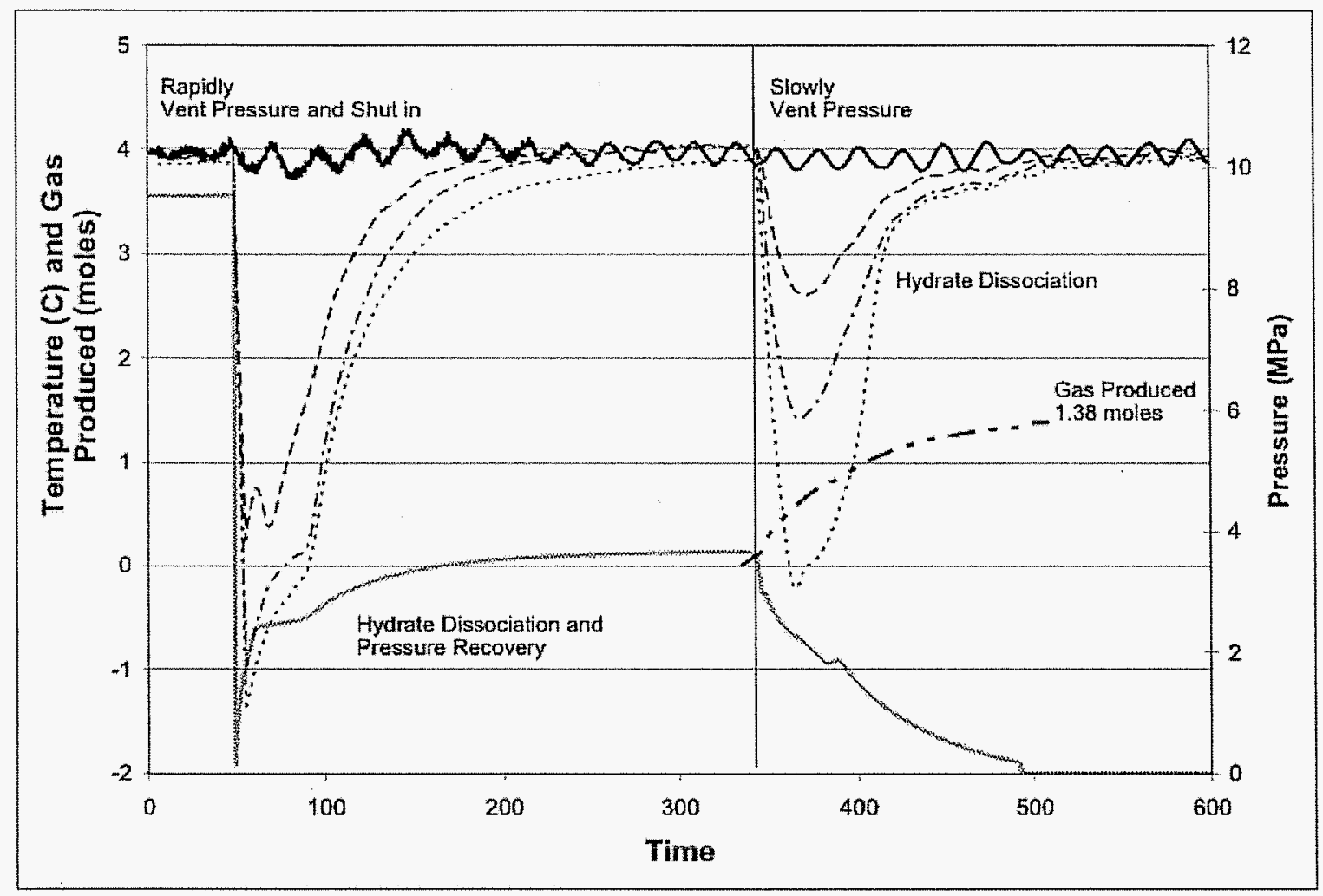

Figure 6. 